\title{
IS SERUM TOTAL CHOLESTEROL ADEQUATELY MONITORED AND TREATED IN PATIENTS WITH A HISTORY OF MYOCARDIAL INFARCTION?
}

\author{
N Almond, GP Registrar \\ Queen Square Surgery, Lancaster
}

\section{INTRODUCTION}

Elevated serum cholesterol is an important risk factor in the morbidity and mortality associated with coronary artery disease (CAD). The Standing Medical Advisory Committee (SMAC) has recently issued guidelines on the use of statins in the treatment of hypercholesterolaemia in patients with established CAD and in those at risk of developing CAD The guidelines draw evidence from several landmark studies illustrating the value of statin therapy in both primary and secondary prevention of CAD. The SMAC document provided the stimulus to audit the patients in the Queen Square practice at high risk, who had already experienced an infarct. The objective was to see how closely the practice observed the existing practice protocols and to make recommendations for changes in line with the latest clinical evidence.

One of the first major studies was the Scandinavian Simvastatin Survival Study (4S) which showed that during the five year study period simvastatin therapy reduced the risk of coronary mortality by $42 \%$ in patients with a previous history of angina or myocardial infarction ${ }^{(2)}$. The study involved a total of 4444 patients. Out of these only 353 had a major coronary event whilst on simvastatin, as opposed to 502 patients on placebo. A major coronary event was defined as cardiac death or non-fatal MI. This provided compelling evidence of the value of statin therapy.

The West of Scotland Coronary Prevention Study (WOSCOPS) was a primary prevention study performed on 6595 men who were at high risk of developing CAD but were symptomless at enrolment ${ }^{(3)}$. Treatment with Pravastatin resulted in a $31 \%$ lower incidence of a major coronary event during the five-year follow-up.

Pravastatin was also shown to have a similar benefit in secondary prevention in the Cholesterol and Recurrent Events (CARE) study, another major trial of 4159 patients with established $\mathrm{CAD}^{(4)}$. This showed benefit in treating patients with only moderately raised cholesterol where the mean baseline total cholesterol was $5.4 \mathrm{mmol} / \mathrm{L}$ before treatment. In particular it was found that the CAD risk reduction was maximal when a 10-20\% reduction in low density lipoprotein (LDL) had been achieved, although no benefit was derived from reducing LDL below a baseline of 3.2 $\mathrm{mmol} / \mathrm{L}$. A separate study looking at patients with coronary

This article was printed in Training Update, February 1999, and is reprinted here by kind permission of the editor. artery bypass grafts has suggested that aggressive treatment of $\mathrm{LDL}$ to values lower than $3.2 \mathrm{mmol} / \mathrm{L}$ did reduce progression of atherosclerosis in vein grafts in this group ${ }^{(5)}$.

Statins are safe and generally well-tolerated drugs. They act by competitively inhibiting the HMG CoA reductase enzyme involved in cholesterol synthesis in the liver. They should be used with caution in patients with a history of liver disease or high alcohol intake, and biochemical monitoring of liver function should be undertaken in these patients. Treatment should be discontinued if serum transaminases rise to greater than three times the upper limit of normal. Another rare but major side effect is reversible myositis and patients should be told to report unexplained muscle pain. If the creatine kinase rises to greater than ten times the upper limit of normal, treatment should be discontinued.

Treatment and monitoring of hypercholesterolaemia is readily achievable in a primary care setting. There is great potential for statin therapy to make a significant impact on coronary morbidity and mortality in 'at risk' patients. The Sheffield table ${ }^{(1)}$ allows a risk assessment to be made using factors in the history such as co-existing diabetes or hypertension. One priority group which should be targeted for treatment of raised cholesterol is those with a history of previous MI or angina. Clearly the cost implications of statin treatment in such a large population are important. The monthly cost of treating one patient ranges from $£ 12-£ 94$ according to the dose and manufacturer (Table 1). These extra costs must be offset against the savings made by reducing future coronary events. It should be noted that the cheapest drug is not necessarily the most cost effective because of differences in efficacy.

\begin{tabular}{|lccc|}
\hline drug dose & $10 \mathrm{mg}$ & $20 \mathrm{mg}$ & $40 \mathrm{mg}$ \\
Fluvastatin & - & $£ 14.90$ & $£ 15.90$ \\
Simvastatin & $£ 18.29$ & $£ 31.09$ & $£ 47.04$ \\
Pravastatin & $£ 16.18$ & $£ 31.09$ & $£ 46.48$ \\
Atorvastatin & $£ 18.88$ & $£ 30.60$ & $£ 47.04$ \\
Cerivastatin & $£ 12.95$ & $£ 17.35$ & $£ 18.20$ \\
& $(100 \mathrm{mcg})$ & $(200 \mathrm{mcg})$ & $(300 \mathrm{mcg})$ \\
\hline
\end{tabular}

Table 1 Cost of one month of statin therapy per patient

The importance of raised cholesterol as a risk factor in established CAD is undisputed. The aim of this audit was to examine the monitoring and treatment measures currently applied within the practice to a group of patients with a documented history of MI. The existing practice protocols have been superseded by the more recent SMAC guidelines 
and it was felt to be important to assess how the practice is currently performing with a view to applying the SMAC recommendations soon. The audit focused on MI patients in particular as these are a readily identifiable group although the SMAC guidelines also apply to other priority groups which may warrant further audit in the future.

\section{CRITERIA AND STANDARDS}

All the following audit criteria apply to patients within the age range 35-69 years and with a diagnosis of myocardial infarction (MI) at any time in the past. These patients are identified in the SMAC guidelines as a priority group and most of the clinical trial data relates to this age range. Four specific audit criteria were selected and the standards applied were chosen after discussion at a practice meeting prior to analysis of the data.

Criteria 1 - patients with a previous record of MI should have a documented total cholesterol (TC) value Std $100 \%$

Criteria 2 - Diet advice or statin therapy should be given if total cholesterol is greater than $5.2 \mathrm{mmol} / \mathrm{L}$

Std $100 \%$

Criteria 3 - If given diet advice only, total cholesterol should be rechecked within six months to check satisfactory response

Std $90 \%$

Criteria 4 - if post treatment cholesterol is still greater than $5.2 \mathrm{mmol} / \mathrm{L}$ following at least four weeks on statin therapy the dose should be increased if tolerated.

Std $90 \%$

The above criteria were chosen after reviewing the existing practice protocol for treating hyperlipidaemia which was based on recommendations from the British Hyperlipidaemia Association. The threshold value for treating raised total cholesterol in this protocol was $5.2 \mathrm{mmol} / \mathrm{L}$ which is higher than the latest SMAC guidelines of $4.8 \mathrm{mmol} / \mathrm{l}$ in priority patients. It did not, however, seem reasonable to apply criteria from the SMAC guidelines at this stage as the advice was only distributed in summer 1997 although the evidence had been available two years earlier. In addition our existing guidelines did not advocate specific use of LDL cholesterol and so total cholesterol was chosen as the criteria for this audit.

It seems reasonable to set a high standard for the measurement of cholesterol value and almost certainly this is now done as a matter of course following an MI. There may be occasional exceptions such as coexisting terminal disease where it is deemed inappropriate. To give diet advice following an $\mathrm{MI}$ is a minimum standard for all patients in whom cholesterol measurement is appropriate. With the latest clinical evidence, especially that from the CARE trial ${ }^{(4)}$, it could be argued that all MI patients should be given statin treatment as a minimum standard. It is certainly reasonable to apply a standard of $100 \%$ in criteria 2 .

The standards for criteria 3 and 4 were more difficult to set although after discussion it was deemed important that the standards should be high. It is not sufficient to give advice or start treatment without following up the outcome in terms of the cholesterol level. In addition if the response is inadequate then it is important to alter treatment appropriately. There is an argument for setting these standards at $100 \%$ as well, but it was felt that with compliance problems and the difficulty of getting patients to attend for regular follow-up then a standard of $90 \%$ was fair.

\section{PREPARATION AND PLANNING}

The initial impetus for this audit was the Standing Medical Advisory Document ${ }^{(1)}$ which had been sent to all registered medical practitioners in the summer of 1997 . The audit began with a review of the major trial literature and our current practice guidelines. The audit was focused to look specifically at patients with a definite history of MI as these are a readily identifiable group for priority treatment. A computer search of 5427 patient records was undertaken to identify all patients in the age range $35-69$ years with a past history of myocardial infarction at any time up to $30 / 11 / 97$. One hundred such patient records were identified.

Subsequent computer searches identified whether a total cholesterol value had been recorded for that patient and whether statin treatment had been commenced. If no cholesterol value was found on the computer record a manual check was made of the written notes and hospital letters to identify whether cholesterol had been measured. Similarly, for those patients not receiving statins and yet with a raised cholesterol, each record was inspected by hand for evidence that diet advice had been given by the GP and recorded in the notes.

One problem was that the patient notes were computerised only two years ago and although the transfer of the written summary sheets is now well under way it is possible that some patients with a history of MI may have been missed from the search. The expected incidence for MI is between $1 \%$ and $2 \%$ of the population. One hundred records were identified and out of 5427 patients this gives an incidence of $1.8 \%$. It seem likely that very few patients will have been missed from the audit as a result of not having the diagnosis of MI on the computer summary.

Another problem was being able to identify patients where concurrent disease such as dementia or terminal cancer would make aggressive treatment of cholesterol inappropriate. If the computer summary showed a raised cholesterol for a patient but no apparent treatment prescribed, the records were inspected closely for extenuating factors such as those described above.

\section{RESULTS AND INTERPRETATION OF DATA}

The raw data from the 100 patients are summarised in Table 2 below.

\begin{tabular}{|lr|}
\hline Ml age 35-69 & 100 \\
TC measured & 98 \\
TC currently $>5.2$ & 46 \\
On statin & 52 \\
\hline
\end{tabular}

Table 2 Data summary, number of patients in right hand column

Ninety eight percent of MI patients had their cholesterol measured at some time. Of the two records that could not be found both dated from over twelve years ago and the patients had no current problems with angina and were infrequent attenders.

Of the 52 patients on statin treatment the different statins used were as shown in Figure 1. 


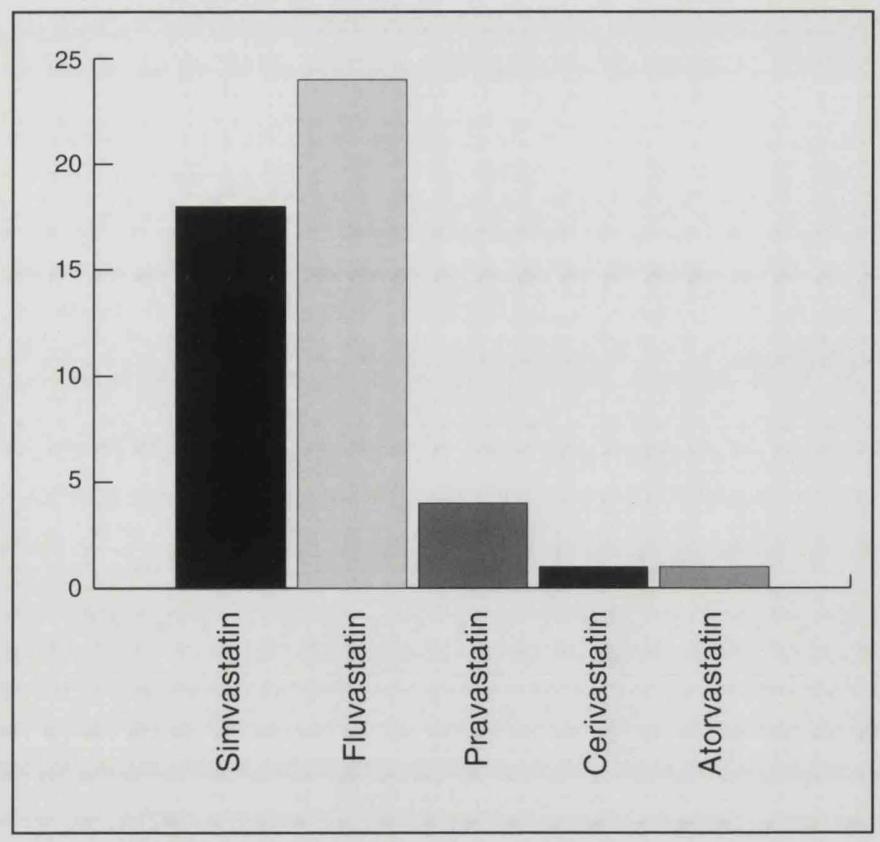

Figure 1 Number of patients on treatment by statin $(n=52)$

Out of the 46 patients with raised cholesterol greater than $5.2 \mathrm{mmol} / \mathrm{L}$ they were given treatment and advice as summarised in Table 3.

\begin{tabular}{|lr|}
\hline On statin therapy & 25 \\
Diet advice only & 11 \\
Fibrate therapy & 1 \\
No recorded advice & 9 \\
\hline
\end{tabular}

Table 3 Summary of treatment of patients with raised cholesterol $(n=46)$.

Of the 98 patients with a recorded cholesterol, 46 had TC values greater than $5.2 \mathrm{mmol} / \mathrm{L}$ as illustrated in Figure 2 .

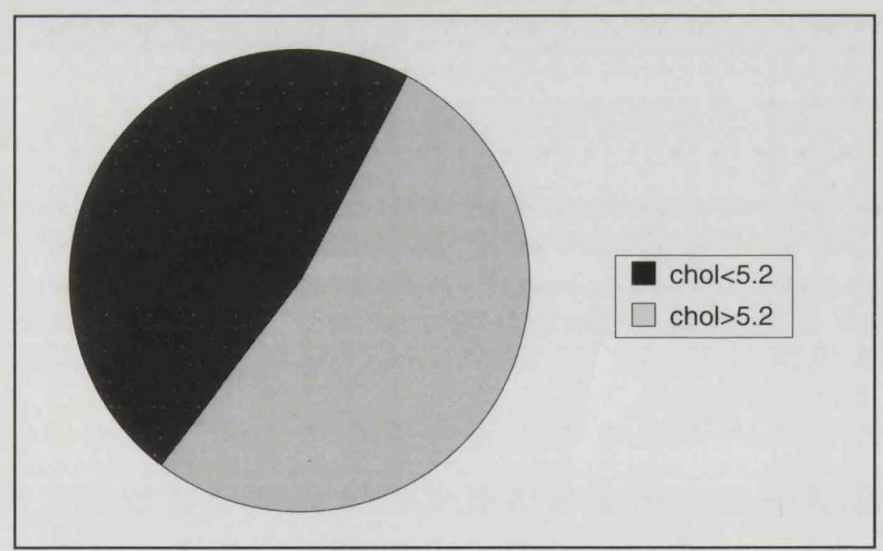

Figure 2 Cholesterol values ( $n=98$ )

As can be seen from these results a significant number of patients did not receive any recorded advice. Within this group of nine it is likely that two are inappropriate for treatment because of concurrent illness. Cholesterol values for the other seven, however, ranged from 5.54 to 7.03 $\mathrm{mmol} / \mathrm{L}$ so they are by no means all borderline cases. It is not possible to say whether advice was given but not recorded in the notes. Of the eleven patients given diet advice only, three may not be appropriate candidates for statin therapy anyway, one because of a drug rash and the other two because of resistance to using medication. Cholesterol values within this group ranged from 5.3 to $7.11 \mathrm{mmol} / \mathrm{L}$.

Of the twenty-five patients with raised cholesterol and currently on statin therapy only ten were either on maximal therapy or had their dose increased in response to the most recent cholesterol value. This result is summarised in Figure

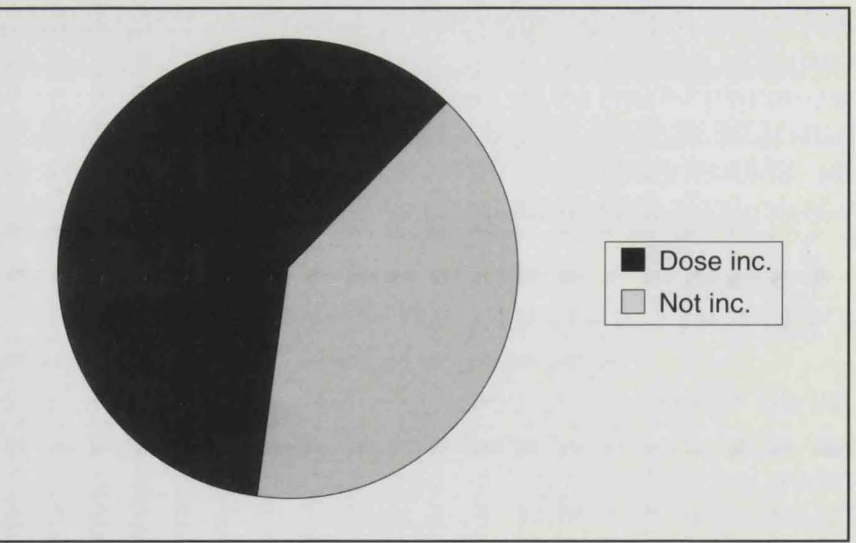

Figure 3 Patients on statin with raised cholesterol $>5.2 \mathrm{mmol} / \mathrm{L}(n=25)$

3. This leaves fifteen patients with raised cholesterol ( 5.25 to $7.51 \mathrm{mmol} / \mathrm{L}$ ) who could have been given an increased dose. Five of these patients did have slightly altered liver biochemistry which may have acted as a deterrent to increasing statin dose, however the transaminase levels were still well below the value of $3 \times$ ULN quoted as significant in the British National Formulary.

\section{STANDARDS ACHIEVED FOR SET CRITERIA}

The results are summarised in Table 3 below.

\begin{tabular}{|lcc|}
\hline Criteria 1-4 & Std Set \% & Std Achieved \% \\
Chol. Measured & 100 & 98 \\
Diet or Statin if $>5.2$ & 100 & 80 \\
Recheck 6 months & 90 & 55 \\
Statin dose incr. if chol $>5.2$ & 90 & 40
\end{tabular}

Table 3 Summary of results

\section{DISCUSSION AND PROPOSALS FOR CHANGE}

Measurement of cholesterol following MI was generally good, but values were not universally entered on the computer record and they are often buried in a mass of paper results. Serum lipids will almost certainly be measured as a matter of course nowadays, following admission to hospital with MI. It is important to ensure that hospital cholesterol measurements are followed up in general practice. It would be helpful to record the baseline cholesterol measurement onto the patient computer record as a routine following receipt of the hospital discharge letter after an admission for MI. Now that patient records are fully computerised any new MI records will certainly have blood results fully entered as well.

The clinical trial evidence has shown important benefits from treating only mild to moderately raised cholesterol values. It can be argued that diet advice alone is not sufficient in the treatment of cholesterol in MI patients. The CARE study in particular showed a significant reduction in MI risk when LDL values were reduced by $10-20 \%$ although no benefit was achieved if the baseline LDL was already $3.2 \mathrm{mmol} / \mathrm{L}$ or less. This is the basis for the new SMAC guidelines. It is proposed to adopt the use of the LDL value in monitoring treatment of our MI patients rather than total cholesterol which also includes the cardioprotective HDL component. This will require slight modification of the disease coding to allow LDL to be stored on the record. 
There is definite scope for improving the number of patients who are prescribed statins. Only just over half of the 46 patients with raised cholesterol were receiving statins although there were only five patients who had reasonable grounds for exception. There is also considerable scope for increasing statin dose in patients whose cholesterol remains raised after starting treatment. In general, follow-up measurement of cholesterol was good but taking action on the value if it was still raised could be improved. The use of the percentage change in the LDL from baseline as the best indicator of adequate response has been suggested and to increase statin dose until a $20 \%$ reduction in LDL is achieved.

Following presentation of the audit at a practice meeting the main proposals for change can be summarised as follows:

All MI patients to have LDL baseline and follow up values recorded on computer

- Diet advice and statin treatment should be started if baseline $\mathrm{LDL}$ is greater than $3.2 \mathrm{mmol} / \mathrm{L}$

Aim for a minimum $20 \%$ reduction in baseline LDL

- Monitor effectiveness of treatment by measuring repeat cholesterol between one and three months after starting or altering statin dose and act on value if not yet achieved $20 \%$ reduction in LDL. Action can be increasing dose if tolerated, or changing to a different statin

One of the main impacts of this audit is that it has shown that our prescribing of statins may need to increase by fifty per cent or more in order to achieve adequate lipid management in our MI patients. In addition, there is a much larger group of 'at risk' patients with angina or CAD risk factors who have been shown to benefit from statin therapy ${ }^{(3)}$.
Future audit work should attempt to include these groups. The cost implications of treating all 'at risk' patients are enormous, as outlined in the introduction (Table 1). However, the costs of not treating these groups may well prove greater, not only in the financial terms of increased hospitalisation but also in the excess morbidity and poor quality of life experienced by patients.

\section{REFERENCES}

1 Standing Medical Advisory Committee: The use of Statins. 11061 HCD 106k Aug 1997

2 Scandinavian Simvastatin Survival Study Group. Randomised trial of cholesterol lowering in 4444 patients with coronary heart disease. Lancet 1994;344:1383-1389

3 Prevention of coronary heart disease with pravastatin in men with hypercholesterolaemia. N Engl J Med 1995; 333:1301-1307

4 The effect of pravastatin on coronary events after myocardial infarction in patients with average cholesterol levels. N Engl J Med 1996;335:001-1009

5 The post-coronary artery bypass graft trial investigators. The effect of aggressive lowering of low-density lipoprotein cholesterol levels and low dose anticoagulation on obstructive changes in saphenous vein coronary artery bypass grafts. N Engl J Med 1997;336:1079-1082

\section{Acknowledgements}

I would like to acknowledge the support of my trainer, Dr DJ Yarnall, and the partners at Queen Square surgery in the preparation of this audit. I would also like to thank my colleagues and trainers in the Lancaster VTS scheme for their helpful discussions. 